\title{
The Metaphor of the Face as an Interface for Communicating Non-Quantitative Information
}

\author{
Simeon J. Simoff \\ University of Sydney, Australia \\ simeon@arch.usyd.edu.au
}

\author{
Fay Sudweeks \\ Murdoch University, Australia \\ sudweeks@murdoch.edu.au
}

\begin{abstract}
In this paper we propose that a metaphor can be used to represent domains that are not easily quantifiable. The metaphor then can be used as an interface to communicate information about those domains between the human and the computer at a cognitive and visual level. We propose a model, which uses the metaphor of a human face as an interface data formatting system for the perception and evaluation of universal aesthetics.
\end{abstract}

\section{Metaphors computers live by}

The title of the introductory section is an adaptation of the title of the seminal work by Lakoff and Johnson (1980), where metaphors depict incomplete parallels between dissimilar ideas or things, emphasising some qualities and suppressing others. For more than two millennia, the metaphor has appealed to scholars interested in language, rhetoric and poetry. In the past few decades, the interest in formal theories of metaphor, which established relationships between its structure, functionality and cognitive nature, has increased in several disciplines including linguistics, philosophy, psychology, education and sciences, and has given birth to the contemporary theory of metaphor (Yu, 1998).

The power of the metaphor has also been employed in the area of human-computer interaction (HCI) and interface design. A popular example is the desktop metaphor proposed by Xerox and popularised by the MacOS operating system. The desktop metaphor takes the office desk as a cognitive and visual framework for organising files on a computer. An icon of a sheet of paper is used to represent a file; icons of folders are used to group computer files together; and an icon of a waste-paper basket is used to dispose of unwanted files. The desktop metaphor is a conceptual and associative metaphor.

Another example of a metaphor in interface design is the "virtual instrument", which was developed to represent a measure of a particular type of physical value. A visual representation of a sound mixer, for example, is used as a metaphor for adjusting the volume of different media channels. The virtual instrument not only provides conceptual and associative information, it also provides a representation of quantifiable information.

Although there is a substantial amount of work about the use of metaphor in interface design, there is still a lack of formalisms and formal approaches in this area. Kuhn et al. (1991) attempted to develop an algebraic approach to the problem, but this approach has not been elaborated. More fruitful research has been conducted by Anderson et al. (1994), who proposed the so-called "pragmatic model" of metaphor mapping based on a representation of the metaphor as a set of features. Anderson et al. defined four groups of features to describe metaphor mapping, which we present in terms of interface design:

- features in the interface, which are supported by the selected metaphor;

- features in the interface, which are not supported by the metaphor;

- features that exist in the metaphor, but are not supported by the interface; 
- features that neither exist in the metaphor nor are supported by the interface.

The first two groups of features have been used by Anderson et al. to investigate the effectiveness of a metaphor in an interface. Defining the last group of features may cause some difficulties. Alty and Knott (1999) offer an interesting formalism, which extends the feature-based model of Anderson et al. to examine relationships, where the "tenor" (the original idea) is transformed (modified) by another idea called "vehicle". Their analysis attempts to cover both system functionality and corresponding interface features. However, Alty and Knott provide neither guidelines for nor examples of how the proposed model-based approach can be implemented. They emphasize that there may be differences between the designer's view of the metaphor and the user's understanding of the same metaphor, which results in difficulties in the interface design. Alty and Knott do not provide a mechanism that guarantees the match between the two views of the same metaphor - they assume that the designer's view and the user's view agree.

While metaphors such as the sound mixer are able to communicate quantifiable information, the use of metaphors for perceiving and evaluating nonquantifiable information is much more problematic. The perception of aesthetics, for example, depends on non-quantifiable factors such as culture, skill and experience, to name but a few. The evaluation of aesthetics is even more problematic. The evaluation is generally left to humans who use a variety of subjective scales that are open to the human failings of error and individual bias. Because computers work with numbers, we need to convert an evaluation category to a number (e.g. "4" on a Likert scale of 1 to 5) in order to communicate with the computer. Usually there are some vague criteria. It is not clear what are the mechanisms of this conversion, i.e. it is usually difficult to formulate precisely why we give "4" for that evaluation category instead of "5" or " 2 ", as shown in Figure 1.
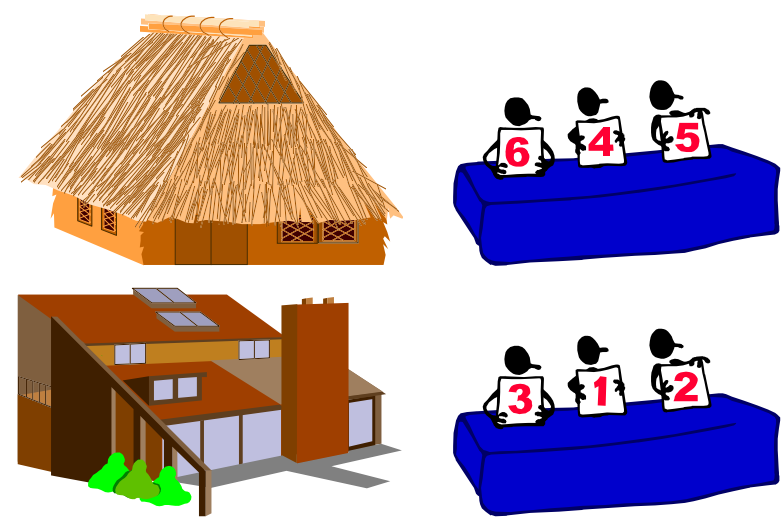

Figure 1. Evaluating the originality of a design

In this paper we propose that consistently formalised metaphor can be used to represent domains that are not easily quantifiable, such as the perception and evaluation of aesthetics. The framework proposed in this paper is based on the idea of visualising instances of metaphors that share configurations, which means that the metaphors have identical basic parts in the same basic arrangement. The instances of such metaphors form an interface between human and computer with which the human is able to communicate ill-defined categories to the computer for processing. The human face is an example of such metaphor. We propose a framework, which uses the metaphor of a face as a computer interface to communicate non-quantitative information between the human and the computer at a cognitive and visual level. In this case the conversion into numbers remains on the side of the computer. The computational representation of the metaphor provides the basis for comparison between different instances of the same metaphor.

In the next section we briefly discuss the issues in communicating aesthetics in human computer interactions.

\section{Aesthetics}

It is well known that the aesthetic quality of a particular art or artifact such as painting, music, design (to name just a few), trigger varying responses in human observers. It is not trivial, however, to relate these responses to particular characteristics of the art or artifact. The notion "aesthetics of design", for example, 
describes those characteristics of the design that are responsible for the appearance and perception of a design artifact, and for that part of the artifact that impacts on our emotional and mental world. In particular, it refers to the responses that indicate the degree of discrimination in perception when confronted with a design. This perception depends on the individual's interpretation, which may arise from emotional responses and/or comparison with previous experiences.

The criteria for aesthetics are usually expressed in the form of ratios between some numeric parameters of the structure of an artifact, whether it is a musical fragment or a building skyline. The perception of aesthetics is verbally described by closely interrelated terms like "style", "taste", "originality" and "beauty". A style refers to designs that have identifiable common characteristics. Personal preferences in style are connected with individual "taste". "Style" and "taste" are connected with the "originality" and individuality of a design, although not everything original is aesthetic. "Beauty" is an even more abstract term. Sometimes it is interpreted as a characteristic of aesthetics, sometimes it is understood as a synonym of aesthetics.

Major difficulties of integrating aesthetics in intelligent computer support for arts and design is communication of aesthetics to machines and the measurement of perception of aesthetics. Even more difficult is the comparison of aesthetics of different types of designs. Can we compare, for example, a building and a "sport style" of a car, saying that the two designs are at the same aesthetic level? Does it mean, that if an individual has assigned 10 to a building and 10 to a car that they produce the same aesthetic perception, the same response to the beauty that they possess?

The possible existence of aesthetics universals has piqued the interest of scholars from many disciplines, including philosophers, psychologists, anthropologists, cultural scientists and sociologists. According to Forge (1973), the existence of a universal human aesthetic is a matter of faith with neither those supporting nor those opposing the notion of a basic or genetic response to certain forms or proportions being able to prove their beliefs.

While there is experimental support for transcultural agreement in the evaluation of aesthetic stimuli (notably Child and Siroto, 1971), there are also critics who point out that it is still not known which visual characteristics elicit a positive response across cultures. As Alland (1989) claims, "While they [the Child and Siroto experiments] suggest that some kind of universal principle (perhaps one concerning form) is operating, we have no way of telling what this principle is." However, there are numerous characteristics of aesthetics that can be regarded as candidates for universal aesthetic principles, such as skill, symmetry, balance, clarity, colour, smoothness, brightness, youthfulness, novelty and fineness (van Damme, 1996).

The experimental evidence of cross-cultural agreement on aesthetic preferences is substantial but not unequivocal. We therefore make the following assumptions:

Assumption 1: Beauty is perceived and evaluated similarly across humans, regardless of the object that exhibits the beauty. In other words, the beauty of a building can generate a similar high-level perception and response as the beauty of a face. For example, there is a high level of agreement panhumans that the Taj Mahal is a beautiful building and that Mona Lisa has a beautiful face. Further, the emotional positive response to both objects is similar. Regardless of whether the object is a building or a face, the reaction caused in humans is of the same polarity.

Assumption 2: Beauty is perceived in holistic manner. In other words, we do not tend to identify the beautiful features first and then, after "summing" all the beautiful features, to judge that an object is beautiful. Consequently, the visual data format should be processed in configural or holistic manner. Psychologists lately tend to agree that when it comes to face perception, a face is more than just the sum of its parts, even that faces are not analysed into separate features at all (Roth and Bruce, 1995, Part III).

\section{The metaphor of a human face as a visual data formatting tool}

The power of face as an object of perception and association has been used in various forms of visual art. Salvador Dali's picture "Abraham Lincoln" is an example of the sensitivity of the human visual data formatting and interpretation system. 
Humans are able to recognise and derive a tremendous amount of knowledge from a human face. Part of this knowledge is concerned with the individual identity of the person and part of the knowledge conveys categorical information about sex, age, race and other personal characteristics. The face also is a window to the inner feelings and moods of the individual through the rich facial musculature that constitutes an elaborate means of expressing emotions.

From a cognitive point of view, the human face has an innate appeal (Fantz, 1970). From a very early age even as young as four days - infants show a preference for looking at representations of faces rather than arbitrary designs or colours (Figure 2).
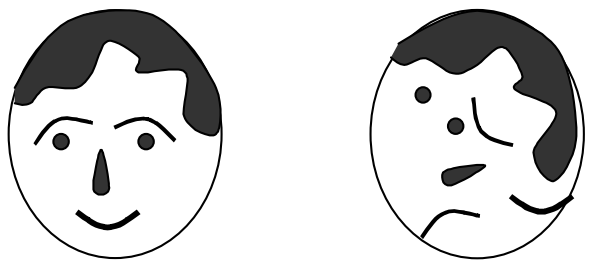

Figure 2. Infants show more preference for looking at the face sketch (left) than the sketch with scrambled facial features (right).

There are two ways of using the face metaphor for data visualisation:

- establish a mapping between model variables and particular facial features;

- establish a mapping between particular states of the face as a whole and particular states of the model.

In the first case we have an example of a classical data analysis/visualisation scheme in which we identify the elements of the model and then assign to each element a particular feature of the visualisation metaphor. Chernoff faces, known in multi-dimensional statistical visualisation, illustrate the idea (Figure 3). Chernoff (1973) introduced a technique of representing $n$-dimensional (originally $n \leq 18$ ) data points by means of faces. Widely divergent facial features are associated with different variables (Figure 4).

Each variable defines the shape of particular facial feature, thus influencing the perception of the face. Any change in the face, i.e. in one or more of these features, when representing a different data point, can be perceived accurately by the observer. The method is based on the assumption that the total facial expression from the face space can be related to the meaning of the data point variable space. An example of a Chernoff face for different data points is shown in Figure 5.

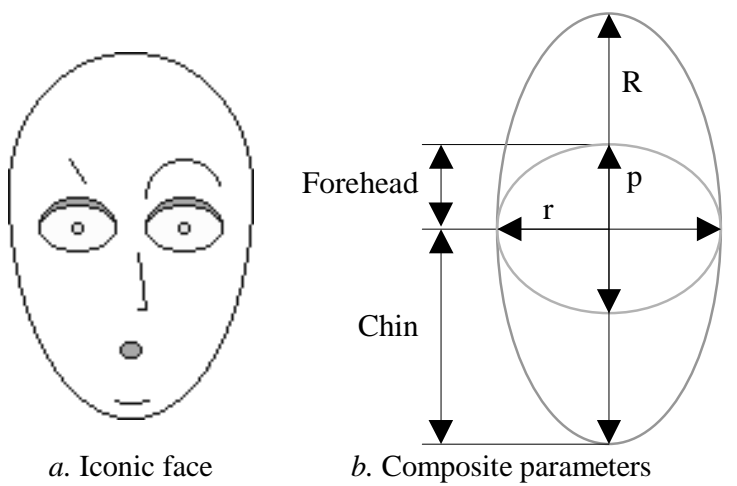

Figure 3. The idea of "feature-variable" representation in Chernoff face

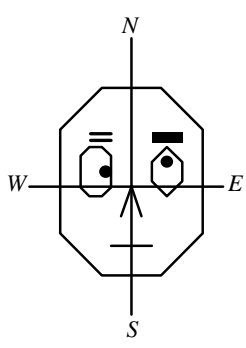

$$
\begin{array}{cl}
\text { Variable } & \text { Feature } \\
1 & \text { NW angle of face } \\
2 & \text { NE angle of face } \\
3 & \text { Left eyebrow } \\
4 & \text { Right eyebrow } \\
5 & \text { Socket of left eye } \\
6 & \text { Pupil of left eye } \\
7 & \text { Socket of right eye } \\
8 & \text { Pupil of right eye } \\
9 & \text { Shape of nose } \\
10 & \text { Shape of mouth } \\
11 & \text { SW angle of face } \\
12 & \text { SE angle of face }
\end{array}
$$

Figure 4. Chernoff type face for 12 dimensional data vectors (adapted from Du Toit et al., 1986).

The example in Figure 5 illustrates the idea of the second way of using the face metaphor for data visualisation. Jones (1996, p. 321) comments on the potential of facial expression: the face could change from happy to sad representing a particular trend in the data. We consider that once a mapping is established (not necessarily of a variable-feature type, as in the case Chernoff faces), then the face can be visually manipulated, generating data which corresponds to the 
perception. In this context we formulate the third assumption, related to the face as a metaphor for communicating non-quantitative information:

Assumption 3: An interactive visual representation of a face can be adjusted by humans to portray categories of beauty which will be evaluated similarly across humans. An underlying mathematical model behind the computer-aided visual representation of a face will generate a corresponding set of numbers (comprising both reals and/or intervals) and there will be a high level of agreement across categories of beauty.

\section{Proposed framework}

As mentioned earlier, to the computer, qualitative categories such as beauty and ugliness expressed in symbolic form mean no more than a string of characters. We propose that the face can offer a mapping between human perception (based on a visualisation of the face) and numerical computing (based on a digital representation and mathematical model behind this representation). The idea is illustrated in Figure 6.

"Perceived beauty" is on the human side. The "Visualised metaphor" establishes the bridge between the human visual/cognition system. "Computable representation" of the metaphor is the parameterised model, of the metaphor, which is used in the computing. Through such an interface we can communicate to the computer the degree of pleasure aroused by a particular aesthetic stimulus by manipulating the model of a face to a state that most accurately represents the experience. The dynamics of the human-computer visual processing is illustrated in Figure 7.

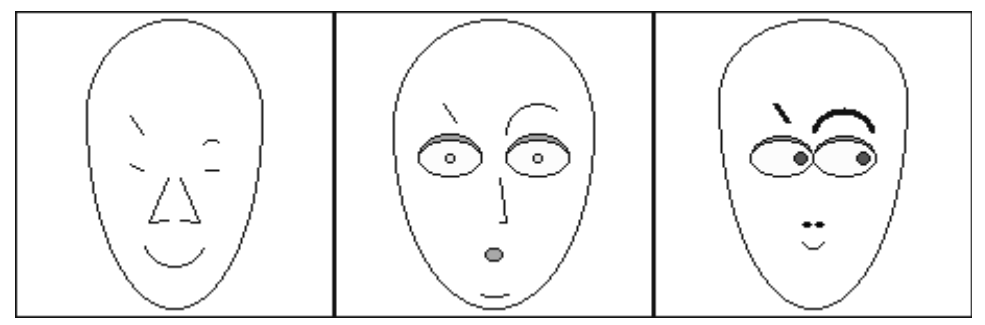

Figure 5. Chernoff faces for different data points.

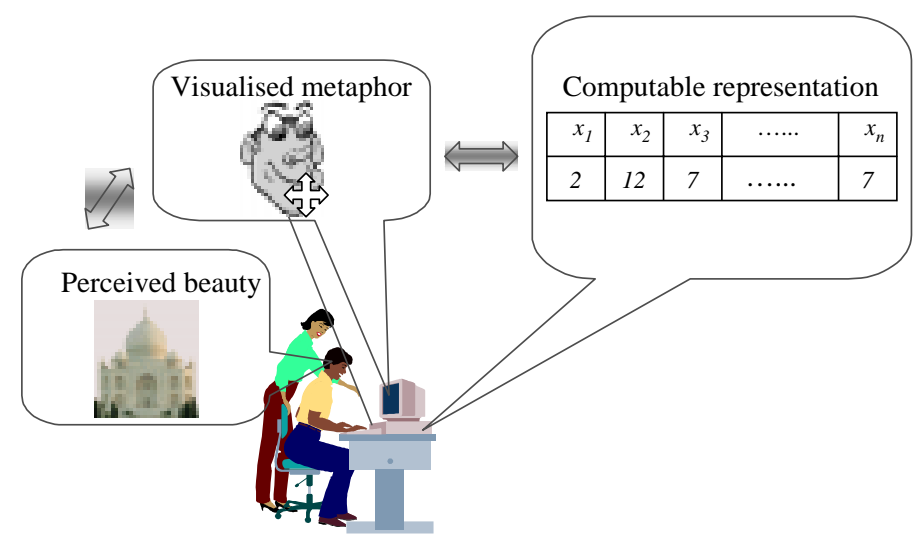

Figure 6. Communicating aesthetic stimulus via the facial metaphor. 


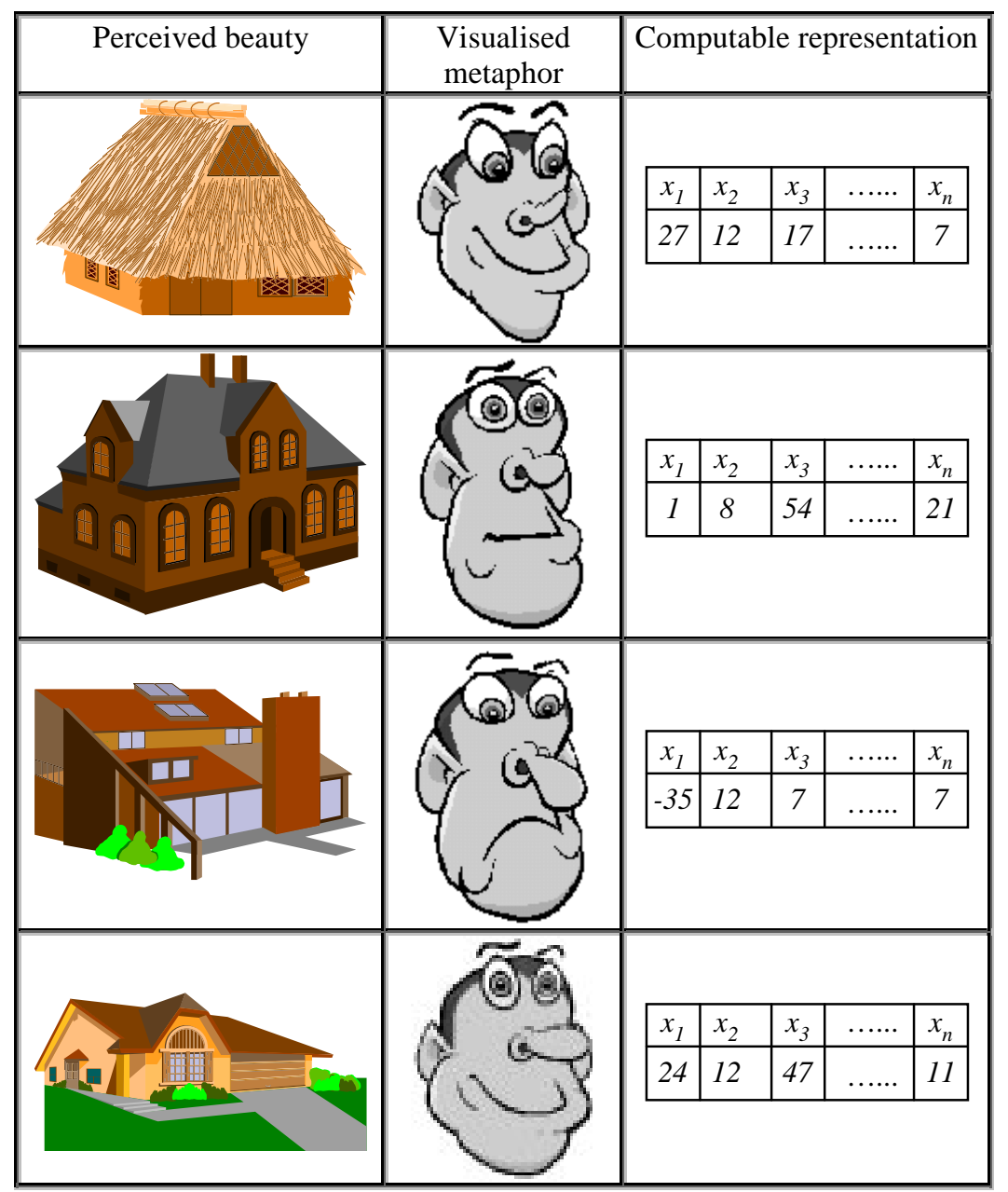

Figure 7. Human-computer visual processing.

From computational point of view the framework utilises both approaches in the use of face metaphor for data visualisation. Each approach is defined by what stands behind the vector $\mathbf{x}$ of the parameters of computable representation. The example in Figure 7 utilises the idea of Chernoff approach - geometrical parameters of identifiable facial features are associated with particular parameters. For example, the shape of mouth in this case is represented by $x_{1}$. However, vector $\mathbf{x}$ can be computed from another facial model, for example, based on the anatomy of facial muscles and skin (Waters, 1992). Parameterised muscle models have been designed to perform complex facial articulations for the creation of synthetic facial expressions based upon biological motivators. As a rule the control of facial articulations is designed to encapsulate the low-level control with high level commands through models of the major muscles and their behavior (Figure 8). 


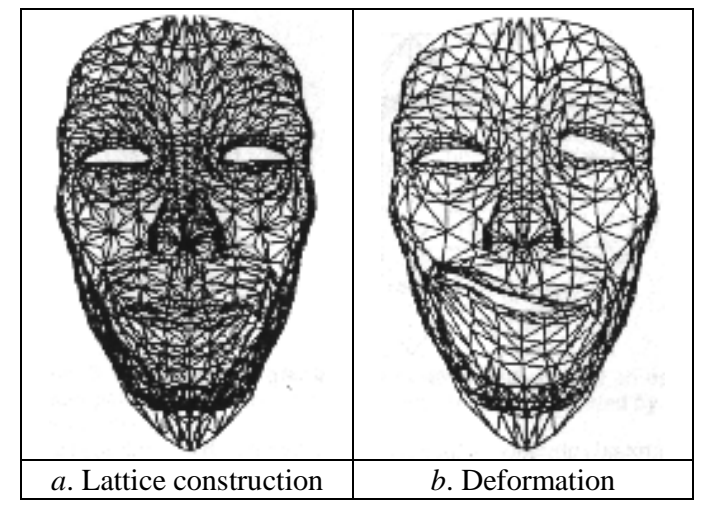

Figure 8. Wireframe facial model, based on paramterised muscle models (adapted from Waters, 1992).
To provide ability to compare resultant faces the framework utilises different similarity and distance measures, which operate over the vector representation of the face space. In this case the human does not have the task to convert his/her own perceptions into numbers. The scenario, shown in Figure 1 translates into sets of faces, whose "values" are compared by computer. An example, of the judgement of a second expert is shown in Figure 9. This also illustrates the potential of proposed framework for building interfaces for computermediated expert judgements systems.

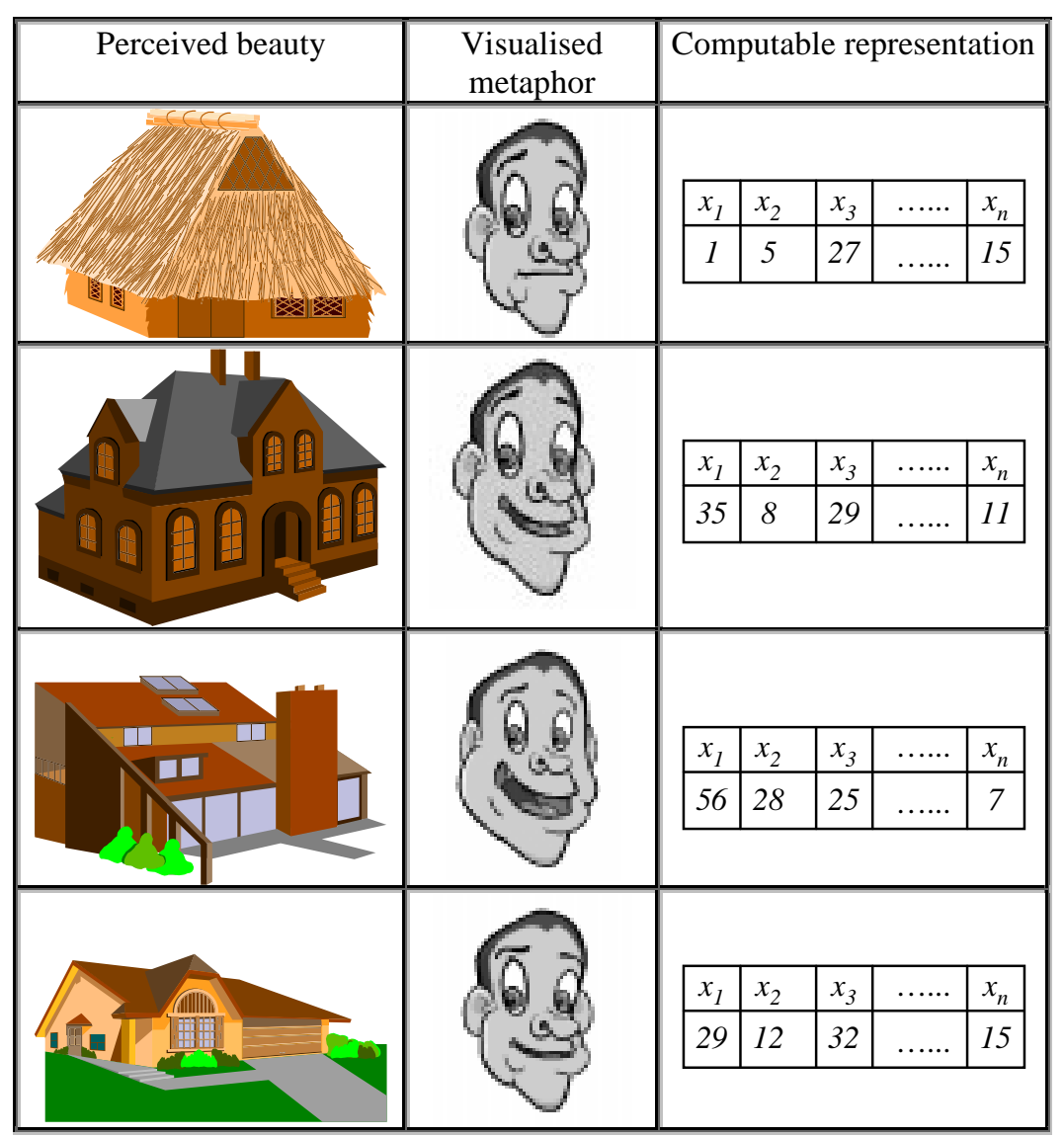

Figure 9. Different experts have different judgements (compare with Figure 7). 


\section{Further investigation}

This paper described the ideas and initial assumptions behind the use of facial metaphor as an interface for communicating aesthetics in human computer visual processing. The application of our model will require the use of high-fidelity 3D interactive facial representations. The research project includes experimentation with individuals who will rank design stimuli according to their perceived aesthetic value and manipulate the interactive $3 \mathrm{D}$ face representation to match their aesthetic experience. The results will then be compared with the computed face vectors.

\section{References}

Alland, A. (1989). Affect and aesthetics in human evolution, Journal of Aesthetics and Art Criticism, 97(1), 1-14.

Alty, J. L. and Knott, R. P. (1999). Metaphor and humancomputer interaction: A model based approach. In Ch. L. Nehaniv (ed.), Computation for Metaphors, Analogy and Agents, Springer, Heidelberg, pp. 307-321.

Anderson, B., Smyth, M., Knott, R. P., Bergan, M., Bergan, J., and Alty, J.L.(1994), Minimising conceptual baggage: Making choices about metaphor. In G. Cockton, S. Draper and G. Weir (eds), ...

Chernoff, H. (1973). Using faces to represent points in Kdimensional space graphically. Journal of American Statistical Association, 68, 361-368.

Child, I. L. and Siroto, L. (1971). Bakwele and American aesthetics evaluations compared, in C. F. Jopling (ed.), Art and Aesthetics in Primitive Societies: A Critical Anthology, E. P. Dutton, New York, pp. 271-89.

Du Toit, S. H. C., Steyn, A. G. W. and Stumpf, R. H. (1986). Graphical Exploratory Data Analysis, Springer, Heidelberg.

Fantz, R. L. (1961). The origin of form perception, Scientific American, 204, 66-72.

Forge, A. (1973). Introduction, in A. Forge (ed.), Primitive Art and Society, Oxford University Press, London, pp. xiii-xxii.

Jones, C. V. (1996). Visualization and Optimization, Kluwer Academic Publishers, Dordrecht.
Kuhn, W., Jackson, J. P. and Frank, A. U. (1991). Specifying metaphors algebraically, SIGCHI Bulletin, 32(1), 58-60.

Lakoff, G. and Johnson, M. (1980). Metaphors We Live By, University of Chicago Press, Chicago.

Van Damme, W. (1996). Beauty in Context: Towards an Anthropological Approach to Aesthetics, E. J. Brill, Leiden.

Waters, K. (1992). Modeling three-dimensional facial expressions. In V. Bruce and M. Burton (eds), Processing Images of Faces. Ablex Publishing Corporation, Norwood, NJ, pp. 202-227.

Yu, N. (1998). The Contemporary Theory of Metaphor: A perspective from Chinese. John Benjamins Publihing Co., Amsterdam. 